Colégio Politécnico da UFSM, Santa Maria - RS

Revista de Gestão e Organizações Cooperativas - RGC

RGC - Santa Maria, RS, v.2, n.3, jan./jun. 2015

ISSN: 2359-0432

DOI: $10.5902 / 2359043216336$

\title{
Evitar Divulgar Perdas: Foi Uma Estratégia Utilizada na Última Década pelas Cooperativas de Crédito Filiadas ao Sicredi?*
}

\author{
Avoiding to report Losses: Was it a strategy used by Credit Unions affiliated to Sicredi in the last \\ decade $^{*}$ ?
}

\begin{abstract}
Resumo:
Este estudo avaliou se as cooperativas de crédito filiadas ao Sistema de Crédito Cooperativo (SICREDI) utilizaram da estratégia de gerenciamento de resultados para evitar divulgar perdas. O tema de gerenciamento de resultados se apresenta eminente tanto na literatura internacional quanto na nacional, e se mostra ainda mais notável no caso das instituições financeiras, já que o gerenciamento de resultados nestas pode impactar negativamente toda a estrutura do sistema financeiro. A amostra foi composta por 118 cooperativas singulares, perfazendo um total de 99,16\% da população no período de 2001 a 2010. Os resultados indicaram que há evidências de gerenciamento de resultados para não divulgar perdas nas cooperativas filiadas ao Sicredi, em períodos semestrais, e especialmente em periodos anuais. Notou-se que $76 \%$ destas cooperativas buscaram evitar divulgar resultados negativos, pois os resultados, divididos pelo volume de operações de crédito, concentram-se em torno do resultado zero, na faixa positiva do histograma. Os resultados desta pesquisa corroboram com a percepção dos analistas do Banco Central do Brasil, e com os resultados para o Sicoob encontrados por Maia et al. (2013).
\end{abstract}

Palavras-chave: Gerenciamento de Resultados, Evitar Divulgar Perdas, Cooperativas de Crédito, Sicredi.

\begin{abstract}
:
This study evaluated whether credit unions affiliated to the SICREDI Cooperative Credit System used earnings management as a strategy to avoid disclosing losses. Earnings management has emerged as a relevant issue the international and national literature, and is even more striking in the case of financial institutions, since its consequences can negatively impact the entire structure of the financial system. The sample was composed of 118 individual credit unions, covering 99.16\% of the population from 2001 to 2010. The results indicated that there is evidence of earnings management, related to not report losses in credit unions affiliated to SICREDI in half year reports, and especially in annual reports. It was also noted that $76 \%$ of these cooperatives sought to avoid disclosing negative results since the results divided by the volume of loans are concentrated around zero in the positive range of the histogram. The results of this study corroborate the former perception of Brazil's Central Bank analysts, and the results for the Sicoob system found by Maia et al. (2013).
\end{abstract}

Keywords: Earnings Management, Avoid Report Losses, Credit Unions, Sicredi.

Recebido: 01/12/2014 Aceito: 15/04/2015

Valéria Gama Fully Bressan ${ }^{1}$, Aureliano Angel Bressan² e José Marcos da Silva Júnior ${ }^{3}$

${ }^{1}$ Universidade Federal de Minas Gerais, Doutorado em Economia Aplicada - valeria.fully@gmail.com

${ }^{2}$ Universidade Federal de Minas Gerais, Doutorado em Economia Aplicada - bressan@ face.ufmg.br

${ }^{3}$ Universidade Federal de Minas Gerais, Graduando em Controladoria e Finanças - silvajunior.jm@gmail.com 


\section{Introdução}

$\mathrm{O}$ cooperativismo de crédito é um importante instrumento de inclusão no sistema financeiro, já consolidado no ambiente internacional e possui relevante papel no cenário econômico do país, ao desempenhar uma importante função social, que se constitui em efetuar a intermediação financeira entre os cooperados, que proveem tanto a demanda quanto a oferta de recursos.

No ano de 2014, enquanto o Sistema Financeiro Nacional (SFN) cresceu 2,6\% no volume de depósitos, as cooperativas de crédito cresceram 20,5\%, seguida pela Caixa Econômica Federal, que cresceu $11,6 \%$ no ano, e pelas demais instituições financeiras (IFs). Com relação às operações de crédito, o SFN cresceu 7,9\% em 2014 e as instituições integrantes do cooperativismo cresceram $12,9 \%$, atrás apenas da Caixa Econômica Federal que cresceu 17,1\% no período. Ainda com relação aos depósitos, a maior parte das IFs apresentou redução nos valores captados entre junho e setembro de 2014, enquanto que as cooperativas aumentaram significativamente o valor captado. Destaque-se ainda para o patrimônio líquido das cooperativas, praticamente idêntico ao da Caixa Federal, demonstrando o alto grau de capitalização dessas cooperativas (PORTAL DO COOPERATIVISMO DE CRÉDITO, 2014b).

Adicionalmente, no cenário nacional, de acordo com dados de junho de 2014, existem 1.150 cooperativas de crédito que administravam ativos totais na ordem de R\$ 138 bilhões. Somando-se a este valor ainda os ativos do Banco SICREDI S.A. e do BANCOOB tem-se ativos totais de R $\$ 186$ bilhões no ramo crédito, representando uma participação de mercado de $2,71 \%$ no total de ativos do mercado financeiro brasileiro e dando às cooperativas de crédito a $6^{\mathrm{a}}$ posição no ranking das maiores instituições financeiras do país. As operações de crédito totais atingiram $\mathrm{R} \$ 80$ bilhões em junho/2014 representando $2,78 \%$ do total do SFN e os depósitos de R $\$ 91$ bilhões representavam 4,73\% (PORTAL DO COOPERATIVISMO DE CRÉDITO, 2014a).

$\mathrm{O}$ tema de gerenciamento de resultados, ou earnings management, se apresenta eminente tanto na literatura internacional quanto na nacional, e tem grande importância no que diz respeito às instituições financeiras. Evidências de que os grandes bancos atuantes no Brasil gerenciam seus resultados contábeis foram encontradas por Goulart (2007), Xavier (2007) e Dantas et al. (2013), e estes estudos salientam ainda, no caso de instituições financeiras, que o gerenciamento de resultados pode impactar negativamente toda a estrutura do sistema financeiro. No caso das cooperativas de crédito brasileiras, o estudo de Maia et al. (2013) constatou que as cooperativas de crédito filiadas ao Sicoob utilizaram da estratégia de gerenciamento de resultados para evitar divulgar perdas e para suavizar os resultados.

O presente estudo pretende contribuir para a discussão da temática do gerenciamento de resultados aplicada às cooperativas de crédito brasileiras filiadas ao SICREDI, como proposto por Maia et al. (2013). Este sistema, conforme dados divulgados no Portal do Cooperativismo de Crédito (2014c), é composto por um banco cooperativo, uma confederação, quatro cooperativas centrais, 99 cooperativas singulares, atuando em onze estados brasileiros (Goiás, Mato Grosso, Mato Grosso do Sul, Pará, Paraná, Rondônia, Rio de Janeiro, Rio Grande do Sul, Santa Catarina, São Paulo e Tocantins) com mais de mil pontos de atendimento.

No âmbito internacional, estudos como o de Hillier et al. (2008) e Brown e Davis (2008) abordaram a questão de práticas de gerenciamento de resultados aplicadas às cooperativas de crédito australianas. Os autores encontraram resultados divergentes no que tange à conclusão a respeito da utilização, por parte das instituições financeiras, de práticas contábeis para gerenciamento de resultados.

Um aspecto específico da realidade brasileira, conforme é exposto por Maia et al. (2013), é que as cooperativas de crédito não têm acesso à captação de recursos via mercado de ações, entretanto, a adequação mínima de capital prevista na legislação vigente pode ser fator motivacional para a prática de gerenciamento de resultados, já que o capital social das cooperativas de crédito é formado essencialmente por quotas-partes dos associados.

As cooperativas de crédito têm, ainda, o seu desempenho econômico perante o quadro social como tema sensível e, de acordo com Maia et al. (2013), este desempenho pode resumir para o associado à eficiência dos gestores e da própria instituição financeira, para a qual a credibilidade é essencial. Resultados negativos ou que oscilam muito podem denotar situação de risco.

As cooperativas de crédito poderiam ser levadas também a gerenciar seus resultados de forma a sua- 
vizar os mesmos, ou a evitar divulgar perdas, ainda que dentro da legislação, mas fazendo uso da discricionariedade sobre certas contas contábeis para evitar sinalizar uma situação de mau desempenho econômico. Conforme Ventura et al. (2009), o instituto da distribuição das sobras líquidas do exercício pelas cooperativas contribui para gerar pressões internas por eficiência e orientação pela maximização do resultado econômico. Isso se dá, segundo os autores, por haver apropriação do resultado positivo pelo associado, de forma individual.

Sendo assim, o objetivo geral deste trabalho foi verificar se há ocorrência de práticas de gerenciamento de resultados contábeis para evitar divulgar perdas em cooperativas de crédito no Brasil vinculadas ao Sistema de Crédito Cooperativo (SICREDI) e teve-se como base o intervalo temporal compreendido entre 2001 e 2010. Desta forma, a amostra compreenderá o sistema cooperativista de crédito com maior representatividade relativa em termos do volume de operações de crédito e total de depósitos em atuação no país.

O presente artigo está estruturado da seguinte forma. A seção 2 apresenta uma revisão da literatura sobre estudos que tratam de gerenciamento de resultados e sua relação com cooperativas de crédito. A seção 3 apresenta os procedimentos metodológicos e a seção 4 analisa os resultados obtidos. A seção 5 apresenta as considerações finais, e por fim, na seção 6 têm-se as referências utilizadas para embasar a realização desta pesquisa.

\section{Referencial Teórico}

\subsection{Gerenciamento de Resultados}

Segundo Martinez (2013), o gerenciamento de resultados é um tema eminente de estudos no Brasil. A origem das investigações sobre o assunto tem fundamento na necessidade de se identificar se as escolhas contábeis, que são delegadas aos gestores pela regulamentação, são utilizadas de modo a se obter resultados que não representem verdadeiramente a condição financeira e patrimonial da organização (RONEN; YAARI, 2010).

A possibilidade de aumento do valor das firmas e da maximização da função de utilidade dos gestores cria incentivos para que as práticas descritas sejam utilizadas. Sua adoção pode representar um benefício para a organização e credores, dada a amenização dos riscos, representados pela volatilidade dos resultados, mas, também, um malefício caso as informações reportadas no curto prazo não se aproximem dos resultados da empresa no horizonte de longo prazo (HEALEY; WAHLEN, 1999).

$O$ gerenciamento de resultados é definido de uma forma mais ampla por Ronen e Yaari (2010, p. 27) como a coleção de decisões da gerência que resulta em não expor a veracidade dos fatos, mas maximiza o valor dos ganhos como é conhecido pela administração e contabilidade. Ainda segundo os autores, tais gerenciamentos podem ser: benéficos, se têm validade no longo prazo; neutros, se revelam os resultados no curto prazo; e malignos, se não revelam nem a realidade de longo prazo nem a de curto prazo. $\mathrm{O}$ gerenciamento de resultados resulta de tomar decisões sobre produções e/ou investimentos antes dos resultados acontecerem, ou de fazer escolhas contábeis que afetam os ganhos e suas interpretações após os verdadeiros valores serem conhecidos.

Segundo Healey e Wahlen (1999), o gerenciamento de resultados ocorre quando os gestores usam julgamentos nos lançamentos contábeis e estruturam transações para alterar os relatórios, de maneira a enganar os investidores sobre o real desempenho econômico da companhia, ou de modo a influenciar a resposta de contratos que dependem dos resultados apresentados nestes relatórios. Martinez (2001) define que alguns setores, principalmente o financeiro e o de seguros, podem possuir mais incentivos que outros para práticas de gerenciamento.

Matsumoto e Parreira (2007), por sua vez, afirmam que uma das principais causas que levam os gestores a praticarem o gerenciamento de resultados é o fato de os Princípios Contábeis não alcançarem todas as situações e incentivos que os gestores podem obter, não representando esta prática, portanto, fraude.

No Brasil, conforme é explorado por Rodrigues et al. (2007), o gerenciamento de resultados é encarado como um conjunto de práticas utilizadas por contadores e gestores que objetivam alcançar determinado resultado contábil, e são caracterizadas como alterações que não extrapolam os limites legais vigentes no país.

Não necessariamente a utilização da ferramenta decorre do manejo de contas das demonstrações contábeis de resultado, podendo, também, ser con- 
sequência de decisões e atos impactantes diretamente nos fluxos de caixa das empresas, conforme afirmado por Martinez (2001, p. 13).

Gunny (2010) aponta duas tipologias de gerenciamento de resultados, a citar: o gerenciamento de accruals, envolvendo as acumulações e o gerenciamento de resultados reais; envolvendo ações discricionárias dos gestores. Paulo (2007) classifica o gerenciamento de resultados como sendo de três tipos: gerenciamento através de escolhas contábeis; através de decisões operacionais e por meio da manipulação classificatória das demonstrações contábeis.

Rosa e Tiras (2013) afirmam que o uso de benchmarks se transformou em evidencia de gerenciamento de resultados, pois, segundo os autores, é plausível crer que gestores racionais manipulariam as demonstrações financeiras se desejassem atingir um objetivo especifico, um nível de lucro, como o lucro zero ou o lucro do último período, ou mostrar apenas lucros positivos, evitando divulgar perdas. Essa estratégia é conhecida como target earnings e será o foco desta pesquisa. De acordo com Goulart (2007) entre as motivações para evitar divulgar perdas tem-se, por parte das firmas, o interesse em corresponder a determinados benchmarks de resultados do mercado.

\subsubsection{Evidências empíricas de gerenciamento de resultados em instituições financeiras}

Diversos autores têm investigado a presença de gerenciamento de resultados em instituições financeiras. Moyer (1990) analisou uma amostra de 142 bancos norte americanos entre 1981 e 1986, visando verificar a existência de incentivos de gestores de bancos a realizar ajustes contábeis quando o índice de capital se encontrava abaixo do mínimo regulamentar. $\mathrm{O}$ autor relacionou provisões para perdas em empréstimos, empréstimos baixados para prejuízo e o índice mínimo de capital.

Ahmed et al. (1999) agregaram uma nova visão às pesquisas ao investigarem a relação do gerenciamento tanto na perspectiva do capital management quanto do earnings management, concluindo que a gestão de capital superou a gestão de resultados quando analisado o período entre 1987 e 1990, e 1991 e 1995, em que houve alterações nas exigências de mínimos de capital.
A partir da investigação do gerenciamento de resultados em instituições financeiras no Brasil entre 2000 e 2004, em 111 conglomerados financeiros em atuação no Sistema Financeiro Nacional, Zendersky (2005) encontrou resultados que lhe permitiram afirmar que os conglomerados financeiros analisados gerenciam os seus resultados contábeis por meio do componente discricionário das despesas de provisão para créditos de liquidação duvidosa.

Xavier (2007) verificou a existência de indícios de que os vinte maiores bancos brasileiros utilizam gerenciamento de resultados. Analisou se os bancos utilizaram ágio em investimentos em controladas e coligadas, operações com títulos e valores mobiliários, provisões para operações de crédito, ou passivos contingentes para gerenciar resultados. Dos 20 bancos estudados, quatro não apresentaram indícios de gerenciamento em nenhuma das áreas e um apresentou indícios nas três áreas em que foram obtidos resultados conclusivos, exceto quanto aos passivos contingentes, para os quais as análises foram inconclusivas.

Goulart (2007) identificou evidências de que grandes bancos brasileiros utilizam a ferramenta de gerenciamento de resultados, avaliando 50 instituições financeiras entre junho de 2002 e dezembro de 2006, por meio de análises de correlação e regressão.

Visando desenvolver e validar um modelo de dois estágios para a identificação da discricionariedade das acumulações com títulos e valores mobiliários pelas instituições bancárias, Dantas et al. (2013) realizaram uma análise dos bancos brasileiros entre o terceiro trimestre de 2002 e o quarto trimestre de 2010, e encontraram evidências de que os bancos utilizaram da discricionariedade para gerenciar os seus respectivos resultados.

Os trabalhos que buscaram verificar a existência de gerenciamento de resultados em instituições financeiras são diversos, ao contrário do que acontece com os que focam em analisar o cooperativismo de crédito, pois esta temática na literatura nacional é recente, com a primeira discussão apresentada no estudo de Maia et. al. (2013) .

$\mathrm{Na}$ literatura internacional, os estudos de Hillier et al. (2008) e Brown e Davis (2008) abordaram a questão da prática de gerenciamento de resultados aplicada às cooperativas de crédito australianas, e tratam especificamente da adequação de capital 
como elemento motivador para ocorrências de gerenciamento de resultados.

Hillier et al. (2008) utilizaram uma amostra composta por 137 cooperativas de crédito, representando quase a metade das 288 cooperativas em operação na Austrália, num período que abrange 31 trimestres entre julho de 1987 e dezembro de 1994. Os autores encontraram evidências de que, durante o período de 12 meses em torno da data que marca a introdução das regras de exigência de capital do Australian Financial Institutions Code (AFIC), as cooperativas em situação de risco empregaram estratégias de contabi-lidade para reduzir a chance de serem colocadas sob supervisão. A estratégia mais frequentemente aplicada foi, segundo os autores, a contabilização discricionária da provisão para operações de crédito.

Brown e Davis (2008) utilizaram uma amostra de dados do período entre 1992 e 2004, também para cooperativas de crédito australianas, após implantação dos requisitos mínimos do índice de capital, e apontam que o retorno sobre ativos será maior em cooperativas de crédito com baixa proporção de capital. No entanto, o estudo indica que as cooperativas são capazes de lidar com seus membros de forma a aumentar sua rentabilidade para atingir as razões de capital exigidas, não sendo constatado o gerenciamento de resultados.

Os resultados encontrados por estes autores para a realidade do cooperativismo australiano foram divergentes no que se refere à conclusão a respeito da utilização, por parte das cooperativas de créditos, de práticas contábeis para gerenciamento de resultados.

No caso brasileiro, o trabalho de Maia et al. (2013) é precursor sobre a temática de gerenciamento de resultados nas cooperativas brasileiras. $\mathrm{O}$ autor testou se as cooperativas de crédito vinculadas ao Sicoob utilizaram da discricionariedade contábil para gerenciar resultados, no período compreendido entre o primeiro trimestre de 2001 e o terceiro trimestre de 2011.

Maia et al. (2013) avaliaram o gerenciamento de resultados por income smoothing, para evitar reportar perdas e o gerenciamento de capital para adequação do patrimônio líquido da instituição financeira em relação aos riscos de seus ativos, de suas operações, bem como de outros riscos envolvendo suas operações e negociações. Essa adequação de capital é tratada internacionalmente pelos Acordos de Basileia e, no Brasil, o Conselho Monetário Nacional e o Banco Central do Brasil são responsáveis por normatizar as regras no país.

No que tange às cooperativas de crédito filiadas ao Sicoob, segundo Maia et al. (2013), a divulgação de resultados negativos ou com grande variabilidade podem denotar situação de risco, remetendo a uma ideia de ineficiência e, até mesmo, de insegurança aos associados das mesmas, que na verdade, são os donos da cooperativa. Por estas razões, as cooperativas de crédito poderiam ser motivadas a gerenciar seus resultados de forma a suavizá-los, ou a evitar reportar perdas, fazendo uso da discricionariedade sobre certas decisões contábeis para evitar revelar uma situação de mau desempenho econômico-financeiro.

As cooperativas podem ser motivadas à prática do gerenciamento de resultados com a tentativa de redução da volatilidade dos resultados, aplicando-se a técnica de suavização dos lucros. Neste sentido, Maia et al. (2013) encontraram evidências empíricas indicando que as cooperativas de crédito vinculadas ao Sicoob utilizam do gerenciamento de resultados nas modalidades: i) income smoothing e ii) para não divulgar perdas. Entretanto, refutaram a hipótese de pesquisa de gerenciamento para adequação de capital.

\section{Procedimentos Metodológicos}

\subsection{Método analítico}

Para avaliar se as cooperativas de crédito filiadas ao Sicredi, no período de 2001 a 2010 fazem uso da estratégia de evitar divulgar perdas, que é uma prática de gerenciamento de resultados contábeis, utilizar-se-á a metodologia de análise de frequência em histogramas, que foi proposta por Burgstahler e Dichev (1997), ao associarem a irregularidade da distribuição de frequência ao gerenciamento de resultados para evitar divulgar perdas. Posteriormente este método foi utilizado por diversos trabalhos sobre gerenciamento de resultados tanto no Brasil, quanto no exterior, tais como: Martinez (2001), Dechow et al. (2003), Moreira (2006), Jacob e Jorgensen (2007), Rodrigues et al. (2007), Goulart (2007), Maia et al. (2013) e Reis (2014).

A análise de frequência em histogramas possibilita verificar se há ou não uma tendência das cooperativas a praticar o gerenciamento de resultados 
com vistas a evitar divulgar perdas. Verifica-se, por meio desta técnica, se as cooperativas possuem maior frequência de resultados pequenos (divididos pelo total de operações de crédito), porém positivos, próximos de resultado igual a zero. A consequência do gerenciamento de resultados para evitar divulgar perdas é que há a tendência de verificação de um ponto de descontinuidade no histograma de distribuição de frequências dos resultados, no ponto de resultado nulo. Conforme esclarece Goulart (2007), assumindo-se uma distribuição normal, há então uma maior concentração de casos de lucros logo acima de zero e frequências menores que esperadas para resultados próximos de zero, porém negativos.

De acordo com Goulart (2007), ao assumir a hipótese de gerenciamento de resultados para evitar divulgar perdas, admite-se que caso a empresa (neste caso, as cooperativas) apure pequeno prejuízo em determinado período, fará uso de práticas de gerenciamento de resultados, com aumento de receitas e/ou redução de despesas, de forma a reverter a situação de pequeno prejuízo e apresentar pequeno lucro. Assim como no estudo de Maia et al. (2013), a situação foi adaptada para as cooperativas, tendo em vista que o associado pode interpretar de forma desproporcionalmente negativa a apresentação de perdas, ainda que pequenas, sendo bem mais favorável a apresentação de pequenas sobras. Este aspecto configura fortes incentivos para evitar a divulgação de perdas.

\subsection{Variável utilizada no estudo}

A variável de interesse se constitui nos 'Resultados' obtidos pelas cooperativas de crédito filiadas ao Sicredi nos balanços semestrais e nos balanços anuais no período de 2001 a 2010. Para eliminar o efeito tamanho, os resultados tanto semestrais, quanto anuais foram ponderados pelas operações de crédito realizadas pelas cooperativas, assim a variável utilizada no estudo foi definida a partir das seguintes contas do Plano Contábil das Instituições Financeiras do Sistema Financeiro Nacional (COSIF):

$$
\text { Resultados }=\frac{(7 \cdot 0.0 .00 .00-9)+(8.0 .0 .00 .00-1)}{1.6 .0 .0 .00 .00-1}
$$

Em que:

7.0.0.00.00- $9=$ Contas de resultados Credoras 8.0.0.00.00-6 $=$ Contas de resultados Devedoras 1.6.0.00.00-1 = Operações de Crédito

\subsection{Amostra e fonte dos dados}

O presente trabalho utilizou da informação de 118 cooperativas de crédito singulares filiadas ao Sicredi no período de 2001 a 2010. Do total de 119 cooperativas singulares pertencentes a este sistema neste período, trabalhou-se com $99,16 \%$ da população, pois uma cooperativa foi excluída devido a informações faltantes. As informações semestrais, neste período, totalizaram 1072 observações, a as informações anuais totalizaram 1087, isso porque quando ocorreu informações faltantes para as contas de resultados credoras, as contas de resultados devedoras ou para as operações de crédito, estas foram retiradas da amostra para evitar distorção no cálculo de índice de resultados sobre operações de crédito. Os dados para realização desta pesquisa foram obtidos no site no Banco Central do Brasil.

\section{Análise e discussão dos resultados}

Inicialmente, para uma melhor compreensão das características descritivas dos resultados das cooperativas de crédito filiadas ao Sicredi, apresenta-se na Tabela 1 a estatística descritiva tanto para os resultados anuais, quanto os semestrais relativos ao período de 2001 a 2010. Nota-se pelo coeficiente de variação que existe uma grande diferença nestas 118 cooperativas filiadas ao Sicredi em termos dos 'resultados sobre as operações de crédito'. É digno de destaque que os resultados semestrais se mostraram com coeficiente de variação de $936 \%$, ao passo que os resultados anuais apresentaram coeficiente de variação de $-351 \%$, ou seja, pode-se afirmar que são cooperativas com muitas diferenças em termos da geração de resultados, mas que quando remete-se aos resultados anuais, neste mesmo grupo de cooperativas, essa dispersão é menor se comparada aos resultados semestrais Este resultado é um primeiro indicativo de que provavelmente há uma preocupação maior, por parte dos gestores, com os resultados anuais que são divulgados. Pode-se inferir que, uma vez que estes resultados são apresentados na primeira assembleia geral ordinária aos cooperados no início do ano subsequente, e, de certa forma, sinalizam como encontra-se a situação financeira da cooperativa a qual são filiados, este torna-se um período cujo gestores se preocupam com os resultados da cooperativa. 
Tabela 1- Estatística Descritiva dos 'Resultados/Operações de crédito' das cooperativas de crédito filiadas ao Sicredi no período de 2001 a 2010.

\begin{tabular}{|c|c|c|}
\hline Estatística Descritiva & $\begin{array}{l}\text { Resultados anuais/operações } \\
\text { de crédito }\end{array}$ & $\begin{array}{l}\text { Resultados Semestrais } \\
\text { /operações de crédito }\end{array}$ \\
\hline Média & $-0,4109$ & 0,0145 \\
\hline Mediana & 0,0214 & 0,0265 \\
\hline Mínimo & $-475,6207$ & $-27,4438$ \\
\hline Máximo & 3,9144 & 32,2674 \\
\hline Desvio-padrão & 14,4276 & 1,3601 \\
\hline Coeficiente de variação & $-35,1149$ & 93,5905 \\
\hline Número de observações & 1087 & 1072 \\
\hline Número de cooperativas & 118 & 118 \\
\hline
\end{tabular}

Fonte: Resultados da pesquisa.

Adicionalmente, para avaliar se as médias dos resultados semestrais e dos resultados anuais se mostraram estatisticamente significativos, avaliouse de acordo com o teste de normalidade de Doornik e Hansen (1994) se estas possuíam distribuição normal. Conforme o resultado deste teste demonstrado na Tabela 2, rejeitou-se a hipótese nula de que a média dos resultados seguem uma distribuição normal, visto que o valor $\mathrm{p}$ da estatística $\mathrm{D}-\mathrm{H}$ foi menor do que $1 \%$. Assim, por não atender o pressuposto da distribuição normal, utilizou-se dos testes não paramétricos para diferença de medianas e o teste de Mann-Whitney para a diferença de distribuição, para avaliar as diferenças entre os 'resultados sobre operações de crédito' anuais e semestrais nas cooperativas filiadas ao Sicredi. A partir dos resultados dos testes não paramétricos, pode-se afirmar que existem diferenças tanto em termos de mediana, quanto de distribuição dos resultados anuais e semestrais das cooperativas filiadas ao Sicredi no período de 2001 a 2010, indicando que há um comportamento distinto entre os resultados semestrais e os anuais. Nota-se pela Tabela 2 que a diferença de mediana é relativamente sutil, porém estatisticamente significativa. Destaca-se que a mediana dos resultados anuais é apenas 0,0051 menor do que mediana dos resultados semestrais, apesar de os dados apresentarem elevado coeficiente de variação.

A partir da análise de frequência em histogramas, buscar-se-á inferir se há ou não uma tendência das cooperativas filiadas ao Sicredi em praticar o gerenciamento de resultados com intuito de evitar divulgar perdas. Desta forma, assume-se a indica- ção de gerenciamento de resultados se as cooperativas possuem maior frequência de resultados pequenos, porém positivos, próximos de resultado igual a zero. Pela análise da Figura 1, nota-se uma grande variação na distribuição de frequência na faixa imediatamente superior a zero, ou seja, na faixa de 0,00 a 0,01 têm-se $14,7 \%$ das frequências no histograma. As faixas de valores negativos próximos de zero apresentam, visivelmente, um número bem menor de observações que as faixas de valores positivos próximos de zero (Figura 1 e Tabela 3 ).

Apesar de haver limitações a esta metodologia, esta é usualmente assumida na literatura como indicativo de gerenciamento para evitar reportar perdas. A situação apresentada na Figura 1 fornece evidências de que, no período analisado com dados anuais, as cooperativas do Sicredi gerenciaram seus resultados de forma a evitar divulgar perdas.

A Tabela 3 sinaliza que 160 observações referentes os 'resultados/operações de crédito' no período de 2001 a 2010, encontravam-se na faixa de 0,00 a 0,01 , ou seja um indicativo de gerenciamento para evitar divulgar perdas, pois os resultados das contas credoras em relação às devedoras foram muito próximos de zero. E aparentemente apesar de computar $14,7 \%$ das observações, nestas 160 observações tem-se a participação de 77 cooperativas das 118 pesquisadas, ou seja, foi uma estratégia utilizada por $65 \%$ das cooperativas filiadas ao Sicredi, e destaca-se que as maiores ocorrências se deram nos anos de 2009 e 2008, anos estes próximos a crise imobiliário financeira que de modo geral afetaram o cenário mundial (Tabela 4). 
Tabela 2- Testes paramétrico e não paramétricos para análise dos Resultados/Operações de crédito das cooperativas de crédito filiadas ao Sicredi no período de 2001 a 2010.

\begin{tabular}{|c|c|c|c|}
\hline Testes & Hipótese Nula & Estatística do Teste & Valor- $\mathbf{p}$ \\
\hline Doornik- Hansen & $\begin{array}{lr}\text { Resultados } & \text { Semestrais } \\
\text { /Operações de Crédito } \\
\text { seguem uma distribuição } \\
\text { normal }\end{array}$ & $\mathrm{D}-\mathrm{H}=5,33 \times 10^{4}$ & 0,0000 \\
\hline Doornik- Hansen & $\begin{array}{lrr}\text { Resultados } & \text { Anuais } \\
\text { /Operações de } & \text { Crédito } \\
\text { seguem uma distribuição } \\
\text { normal }\end{array}$ & $\mathrm{D}-\mathrm{H}=1,63 \times 10^{6}$ & 0,0000 \\
\hline Mediana & $\begin{array}{l}\text { Resultados semestrais } \\
\text { /Operações de Crédito } \\
\text { possui a mesma mediana } \\
\text { dos Resultados Anu- } \\
\text { ais/Operações de Crédito }\end{array}$ & $\begin{array}{l}\text { Pearson } \quad \text { chi2(1) }= \\
16,5447\end{array}$ & 0,0000 \\
\hline Teste de Mann-Whitney & $\begin{array}{lr}\text { Resultados } & \text { semestrais } \\
\text { /Operações de } & \text { Crédito } \\
\text { possuem a mesma distri- } \\
\text { buição dos Resultados } \\
\text { Anuais/Operações de } \\
\text { Crédito }\end{array}$ & $\mathrm{z}=3,321$ & 0,0009 \\
\hline
\end{tabular}

Fonte: Resultados da pesquisa.

Figura 1 - Distribuição de frequência dos resultados anuais dividido pelo volume de operações de crédito das cooperativas de crédito do Sicredi no período de 2001 a 2010

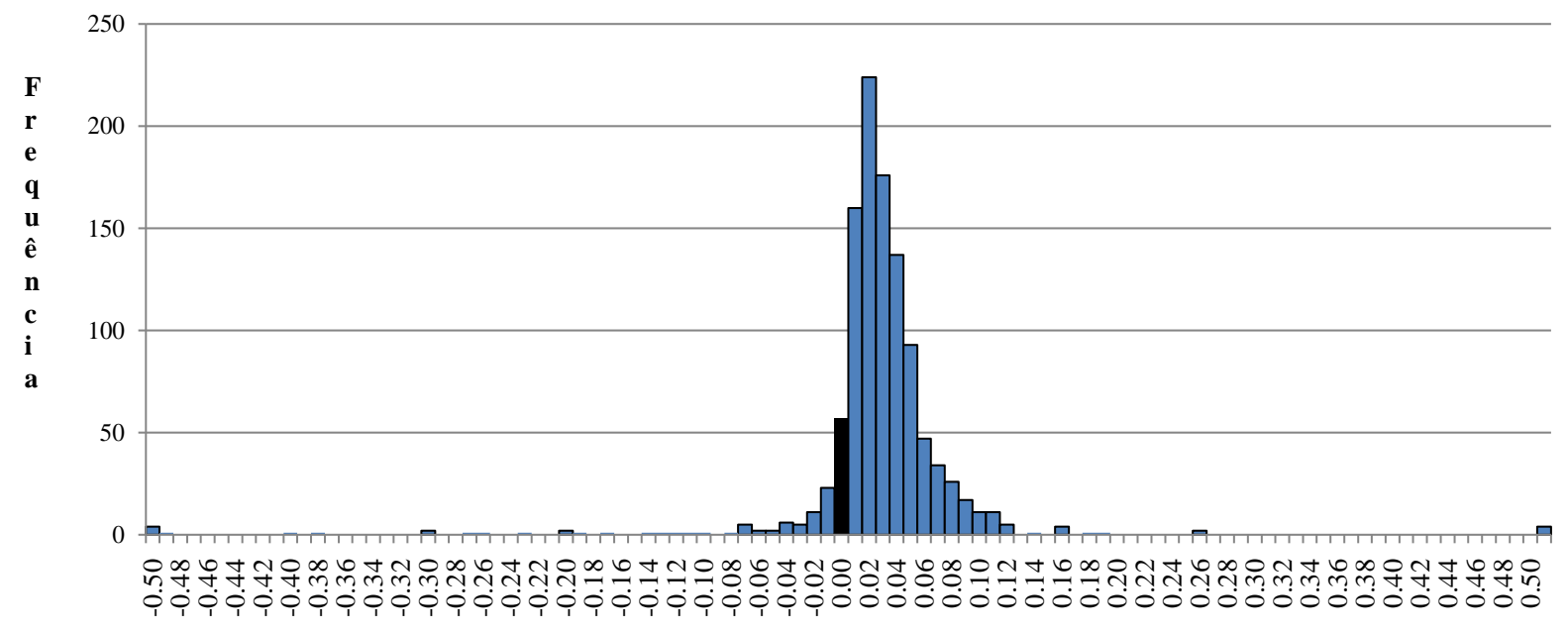

Resultados anuais/Operações de crédito

Fonte: Resultados da Pesquisa. 
Tabela 3- Faixas dos Resultados Anuais/Operações de crédito das cooperativas de crédito filiadas ao Sicredi no período de 2001 a 2010.

\begin{tabular}{|c|c|c|c|}
\hline $\begin{array}{l}\text { Faixas dos Resultados Anuais/Operações de } \\
\text { crédito }\end{array}$ & $\begin{array}{l}\text { Número de } \\
\text { observações }\end{array}$ & Percentual & $\begin{array}{l}\text { Percentual } \\
\text { acumulado }\end{array}$ \\
\hline$-0,50$ a $-0,01$ & 76 & $7,0 \%$ & $7 \%$ \\
\hline$<-0,01$ a 0,00 & 57 & $5,2 \%$ & $12,24 \%$ \\
\hline$<0,00$ a 0,01 & 160 & $14,7 \%$ & $26,95 \%$ \\
\hline$<0,01$ a 0,02 & 224 & $20,6 \%$ & $47,56 \%$ \\
\hline$<0,02$ a 0,03 & 176 & $16,2 \%$ & $63,75 \%$ \\
\hline$<0,03$ a 0,04 & 137 & $12,6 \%$ & $76,36 \%$ \\
\hline$<0,04$ ou superior & 257 & $23,6 \%$ & $100,00 \%$ \\
\hline Total de observações & 1087 & & \\
\hline
\end{tabular}

Fonte: Resultados da Pesquisa

Tabela 4 - Número de ocorrências dos Resultados Anuais/Operações de crédito na faixa maior do que 0,00 a 0,01 por cooperativa de crédito filiada ao Sicredi no período de 2001 a 2010.

\begin{tabular}{|c|c|c|c|c|c|c|c|c|c|c|c|}
\hline Cooperativas & 2001 & 2002 & 2003 & 2004 & 2005 & 2006 & 2007 & 2008 & 2009 & 2010 & Total Geral \\
\hline Coop1 & 1 & 1 & 1 & & & & & & & & $\begin{array}{r}3 \\
\end{array}$ \\
\hline Coop2 & & & & & & & & 1 & & & 1 \\
\hline Coop3 & & & & & & 1 & & & & & 1 \\
\hline Coop4 & & 1 & & & & & & & 1 & & 2 \\
\hline Coop6 & & & & & & & & & 1 & & 1 \\
\hline Coop7 & & & & & & & & & 1 & & 1 \\
\hline Coop8 & & 1 & & & & & & & & & 1 \\
\hline Coop9 & & & 1 & & & & & 1 & & 1 & 3 \\
\hline Coop10 & & & & & & 1 & & & & & 1 \\
\hline Coop11 & & & & & & & & & & 1 & 1 \\
\hline Coop12 & 1 & & & & & & 1 & 1 & & & 3 \\
\hline Coop13 & 1 & & & & & & & 1 & 1 & & 3 \\
\hline Coop14 & & & & & & & & & 1 & 1 & 2 \\
\hline Coop15 & & 1 & & 1 & 1 & & & & & & 3 \\
\hline Coop16 & & & & & & 1 & 1 & & 1 & & 3 \\
\hline Coop17 & & & & & & & & & 1 & & 1 \\
\hline Coop21 & & & & & & & & & & 1 & 1 \\
\hline Coop22 & & & & & & & & 1 & & & 1 \\
\hline Coop23 & & & & & 1 & & & & & & 1 \\
\hline Coop24 & & & & & & 1 & & & 1 & & 2 \\
\hline Coop25 & & & & & & & & & & 1 & 1 \\
\hline Coop26 & & & & & & & & & & 1 & 1 \\
\hline Coop27 & & & & & & & & & & 1 & 1 \\
\hline Coop28 & & & & & & & & & 1 & 1 & 2 \\
\hline Coop29 & & & & & & & 1 & & & & 1 \\
\hline Coop30 & & & & & & & & & 1 & & 1 \\
\hline
\end{tabular}




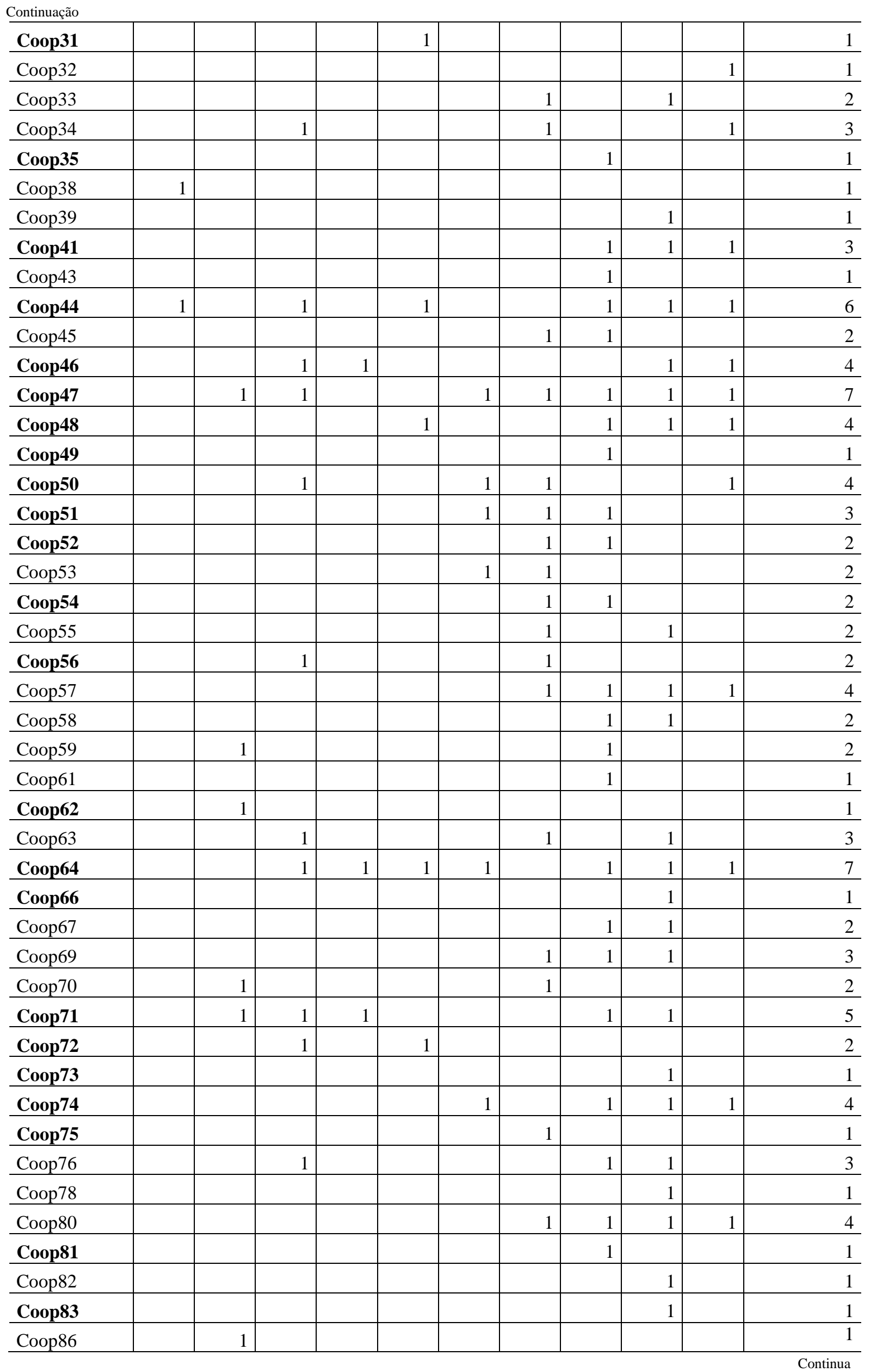




\begin{tabular}{|c|c|c|c|c|c|c|c|c|c|c|c|}
\hline \multicolumn{12}{|c|}{ Continuação } \\
\hline Coop84 & & & & & & & & 1 & & & 1 \\
\hline Coop87 & & & & & & 1 & & 1 & & & 2 \\
\hline Coop88 & & & & & & & & & 1 & & 1 \\
\hline Coop89 & & & 1 & & & & & & 1 & 1 & 3 \\
\hline Coop90 & & 1 & & & & & & 1 & 1 & & 3 \\
\hline Total Geral & 5 & 11 & 14 & 4 & 7 & 11 & 20 & 31 & 36 & 21 & 160 \\
\hline
\end{tabular}

Fonte: Resultados da Pesquisa.

Os resultados deste estudo aplicado ao Sicredi, coadunam com os resultados para o Sicoob realizado por Maia et al. (2013). Estes autores descreveram que o gerenciamento de resultados para evitar divulgar perdas era uma situação esperada pelos técnicos do BACEN, uma vez que anualmente as cooperativas divulgam seus resultados aos associados. Por ocasião das assembleias ordinárias, os resultados são divulgados ao quadro social da cooperativa, ficando o gestor em evidência perante os associados que ele representa. Resultados negativos podem ser aliados a uma percepção de má gestão, prejudicando o status do gestor, além de denotar má situação da cooperativa, o que pode provocar perda de credibilidade perante os clientes, que são, na verdade, os donos da instituição. Uma vez que a credibilidade é um aspecto fundamental para uma instituição financeira, a cooperativa tende a fazer esforços para não divulgar perdas, podendo geren- ciar os resultados de forma a obter pequenas sobras ao invés de pequenas perdas.

Avaliou-se também a distribuição de frequência dos resultados semestrais das cooperativas filiadas ao Sicredi no período 2001 a 2010. A Figura 2 fornece evidências de que as cooperativas filiadas ao Sicredi gerenciaram seus resultados semestrais, mas em menor grau em relação aos resultados anuais, assim como ocorreu para o Sicoob na pesquisa realizada por Maia et al. (2013). Nota-se pela Tabela 5, que para os resultados semestrais apenas $8,7 \%$ da amostra, ou seja, 93 observações encontravam-se na faixa de 0,00 a 0,01. Adicionalmente, o número de cooperativas com os resultados nesta faixa foi de apenas 56 (47\%), indicando que tem-se uma queda quando compara-se com os resultados anuais (Tabela 6).

Figura 2 - Distribuição de frequência dos resultados semestrais dividido pelo volume de operações de crédito das cooperativas de crédito do Sicredi no período de 2001 a 2010

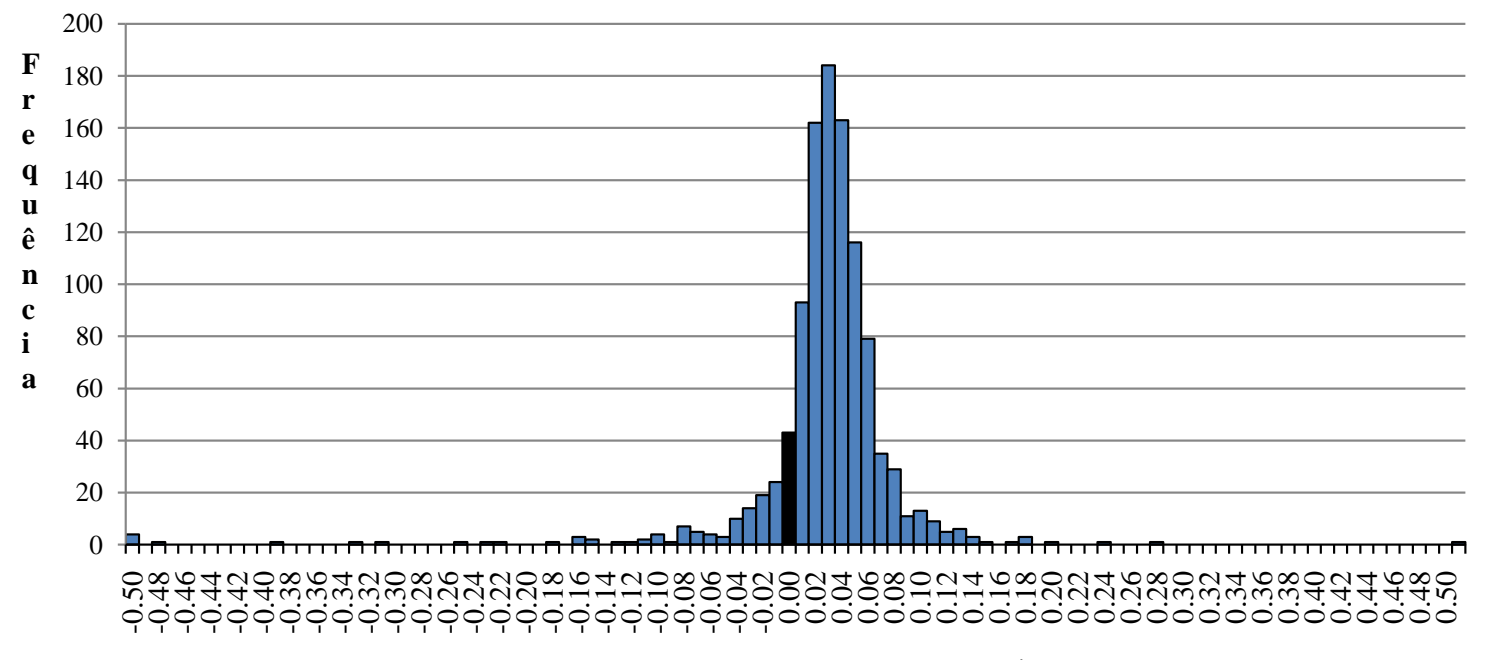

Resultados Semestrais/Operações de crédito 
Tabela 5 - Faixas dos Resultados Semestrais/Operações de crédito das cooperativas de crédito filiadas ao Sicredi no período de 2001 a 2010.

\begin{tabular}{l|r|r|r}
\hline $\begin{array}{l}\text { Faixas dos Resultados Semes- } \\
\text { trais/Operações de crédito }\end{array}$ & $\begin{array}{l}\text { Número de } \\
\text { observações }\end{array}$ & Percentual & $\begin{array}{l}\text { Percentual } \\
\text { acumulado }\end{array}$ \\
\hline$-0,50$ a $-0,01$ & 112 & $10,4 \%$ & $10 \%$ \\
\hline$<-0,01$ a 0,00 & 43 & $4,0 \%$ & $14,46 \%$ \\
\hline$<0,00$ a 0,01 & $\mathbf{9 3}$ & $\mathbf{8 , 7 \%}$ & $23,13 \%$ \\
\hline$<0,01$ a 0,02 & 162 & $15,1 \%$ & $38,25 \%$ \\
\hline$<0,02$ a 0,03 & 184 & $17,2 \%$ & $55,41 \%$ \\
\hline$<0,03$ a 0,04 & 163 & $15,2 \%$ & $70,62 \%$ \\
\hline$<0,04$ ou superior & 315 & $29,4 \%$ & $100,00 \%$ \\
\hline Total de observações & $\mathbf{1 0 7 2}$ & & \\
\hline
\end{tabular}

Fonte: Resultados da pesquisa.

Tabela 6 - Número de ocorrências dos Resultados Semestrais/Operações de crédito na faixa maior do que 0,00 a 0,01 por cooperativa de crédito filiada ao Sicredi no período de 2001 a 2010.

\begin{tabular}{|c|c|c|c|c|c|c|c|c|c|c|c|}
\hline Cooperativas & 2001 & 2002 & 2003 & 2004 & 2005 & 2006 & 2007 & 2008 & 2009 & 2010 & Total Geral \\
\hline Coop1 & & & 1 & & & & & & & & 1 \\
\hline Coop3 & & & & & & & 1 & & & & 1 \\
\hline Coop4 & & & 1 & & & & 1 & & 1 & & 3 \\
\hline Coop5 & & & & 1 & & & & & & & 1 \\
\hline Coop6 & & & & & & & & & 1 & & 1 \\
\hline Coop9 & & & & & & 1 & & 1 & & & 2 \\
\hline Coop10 & & & & & & & & 1 & & & 1 \\
\hline Coop11 & & & & & & & 1 & & & & 1 \\
\hline Coop12 & & & & & & 1 & & 1 & & & 2 \\
\hline Coop14 & & & & 1 & & 1 & & & & & 2 \\
\hline Coop15 & & & & 1 & & 1 & & & & & 2 \\
\hline Coop16 & & & & 1 & & & & 1 & & 1 & 3 \\
\hline Coop17 & & & & & & 1 & & & & & 1 \\
\hline Coop18 & & & 1 & & & & & & & 1 & 2 \\
\hline Coop19 & & & & 1 & & & & & & & 1 \\
\hline Coop20 & & & & & & 1 & & & & & 1 \\
\hline Coop22 & & & & & & & 1 & & & & 1 \\
\hline Coop23 & & & & & & & & 1 & & & 1 \\
\hline Coop24 & & & & & & & & 1 & & & 1 \\
\hline Coop29 & 1 & & & 1 & & 1 & & & & & 3 \\
\hline Coop31 & & 1 & & & & & 1 & & & & 2 \\
\hline Coop35 & & & & & 1 & & & & 1 & 1 & 3 \\
\hline Coop36 & 1 & & & & & & & & & & 1 \\
\hline Coop37 & & & & & & 1 & & & & & 1 \\
\hline Coop40 & & & & & 1 & & 1 & & & & 2 \\
\hline Coop41 & & & & & & & & & & 1 & 1 \\
\hline
\end{tabular}




\begin{tabular}{|c|c|c|c|c|c|c|c|c|c|c|c|}
\hline \multicolumn{12}{|l|}{ Continuação } \\
\hline Coop42 & & & 1 & & & & & & & & 1 \\
\hline Coop44 & & & & 1 & & & & & & & 1 \\
\hline Coop46 & 1 & & & & & & & & 1 & 1 & 3 \\
\hline Coop47 & & & & & & 1 & & & 1 & 1 & 3 \\
\hline Coop48 & & & & 1 & & 1 & & & 1 & 1 & 4 \\
\hline Coop49 & & & & & & & & & & 1 & 1 \\
\hline Coop50 & & & & & & & & 1 & & 1 & 2 \\
\hline Coop51 & & & & & & 1 & 1 & & & & 2 \\
\hline Coop52 & & & & 1 & & & & & & & 1 \\
\hline Coop54 & & & & & & & & & 1 & & 1 \\
\hline Coop56 & & & & & & 1 & & & & & 1 \\
\hline Coop60 & & & & & 1 & & & & & & 1 \\
\hline Coop62 & & & & 1 & 1 & . & & 1 & & 1 & 4 \\
\hline Coop64 & & & & & 1 & 1 & & & 1 & & 3 \\
\hline Coop65 & & & & 1 & & & & & & 1 & 2 \\
\hline Coop66 & & & & & 1 & & 1 & & & & 2 \\
\hline Coop68 & 1 & & & & & & & & & & 1 \\
\hline Coop71 & & & & 1 & 1 & & & & & & 2 \\
\hline Coop72 & & 1 & & & & & & & & & 1 \\
\hline Coop73 & & & & & & & & & & 1 & 1 \\
\hline Coop74 & & & & & & & 1 & & & 1 & 2 \\
\hline Coop75 & & & & 1 & & & & & & & 1 \\
\hline Coop77 & & & & & 1 & & & & & & 1 \\
\hline Coop79 & & & & 1 & & 1 & & 1 & & & 3 \\
\hline Coop81 & & & & & & & 1 & & & & 1 \\
\hline Coop83 & & & & & & & 1 & & & & 1 \\
\hline Coop84 & & & & & & 1 & & & & & 1 \\
\hline Coop85 & 1 & & 1 & & 1 & & & & & & 3 \\
\hline Coop90 & & & & & & 1 & 1 & & & & 2 \\
\hline Total Geral & 5 & 2 & 5 & 14 & 9 & 16 & 12 & 9 & 8 & 13 & 93 \\
\hline
\end{tabular}

Fonte: Resultados da Pesquisa.

Conforme a Tabela 6, das 93 observações tem-se a participação de 56 cooperativas das 118 pesquisadas, ou seja, foi uma estratégia utilizada por $47 \%$ das cooperativas filiadas ao Sicredi, e destaca-se que as maiores ocorrências se deram no de 2006 (Tabela 6), diferentemente dos anos de ocorrência para os resultados anuais.

Assumindo-se a hipótese de gerenciamento de resultados para não divulgar perdas, a comparação entre as duas figuras ( 1 e 2) sugere que é mais relevante para os gestores das cooperativas filiadas ao Sicredi não divulgar perdas anuais do que não reportar perdas semestrais. Os resultados semestrais não são divulgados em assembleia, mas são publi- cados em jornais de circulação regional, na área de atuação da cooperativa. Assim, os resultados das figuras denotam uma maior importância dada pelas cooperativas aos resultados anuais que aos resultados semestrais.

Assim como no estudo de Maia et al. (2013), ressalta-se que por ocasião da divulgação dos resultados anuais, a cooperativa e seus gestores ficam em maior evidência perante o quadro social em relação aos resultados semestrais. Ademais, resultados negativos no primeiro semestre podem ser compensados por resultados positivos no segundo semestre, ou seja, a obtenção de resultados negati- 
vos no primeiro semestre, não implica, necessariamente, em resultados negativos durante o ano.

Adicionalmente, pode-se notar pelas Tabelas $4 \mathrm{e}$ 6 , com as cooperativas sinalizadas em negrito, que 41 cooperativas apresentaram os resultados/operações de crédito próximo de zero tanto para os resultados semestrais, quanto para os resultados anuais, o que representa, aproximadamente, $35 \%$ da população. Do total de cooperativas pesquisadas, ou seja, das 118 cooperativas, 90 cooperativas no período de 2001 a 2010 apresentaram a ocorrência de resultados sobre operações de crédito na faixa de 0,00 a 0,01 , seja para dados semestrais, seja para dados anuais, e estas, representam $76 \%$ das cooperativas filiadas ao Sicredi, sendo assim um indicativo de evitar divulgar perdas, uma estratégia utilizada pelo Sicredi na última década.

\section{Considerações Finais}

O presente estudo teve como objetivo verificar se as cooperativas de crédito filiadas ao Sicredi, na última década, utilizaram da estratégia de gerenciamento de resultados para evitar divulgar perdas, a partir da metodologia de análise de histograma proposta por Burgstahler e Dichev (1997). A amostra foi composta por 118 cooperativas singulares, perfazendo um total de $99,16 \%$ da população.

Os resultados indicaram que há evidências de gerenciamento de resultados para não divulgar perdas, por parte das instituições estudadas, em períodos semestrais, e especialmente em períodos anuais. Notou-se que $76 \%$ das cooperativas filiadas ao $\mathrm{Si}$ credi ou nos resultados semestrais ou nos resultados anuais buscaram evitar divulgar resultados negativos, pois os resultados, divididos pelo volume de operações de crédito, concentram-se em torno do resultado zero, na faixa positiva do histograma. Evidenciado que há uma frequência acima do esperado para resultados próximos de zero, porém positivos e, por outro lado, uma frequência bem menor para resultados próximos de zero, porém negativos.

Assim, como destacado por Maia et al. (2013) embora haja críticas e limitações técnicas quanto à metodologia utilizada para confirmação ou refutação desta última hipótese, a distribuição de frequências de forma "anormal" em torno de zero é usualmente assumida na literatura como indicativo de gerenciamento de resultados para evitar divulgar perdas. Adicionado ao fato de que na perspectiva de analistas do Banco Central do Brasil conforme informado por Maia et al. (2013), a aversão a divulgar perdas foi indicada como a mais esperada de ocorrer no sistema cooperativista de crédito brasileiro. Desta forma, os resultados desta pesquisa corroboram tanto com a perspectiva dos analistas do Bacen, quanto com os resultados para o Sicoob encontrados por Maia et al. (2013).

Novamente, de acordo com Maia et al. (2013), destaca-se que a ocorrência de gerenciamento de resultados de forma a evitar perdas, justifica-se pelo fato de que os resultados das cooperativas são importantes indicadores de sua eficiência. Estes resultados podem servir como indicador de desempenho da cooperativa, como um indicador de qualidade da gestão, o que gera, portanto, forte impacto sobre a reputação dos gestores da cooperativa perante o quadro de associados. Soma-se a isto o fato de que, em se tratando de instituição financeira, a credibilidade é um aspecto essencial. $\mathrm{O}$ fato de que resultados negativos podem ser desproporcionalmente interpretados como má situação também justifica a tendência a fazer com que pequenos resultados negativos tornem-se pequenos resultados positivos, utilizando-se a margem de julgamento sobre as demonstrações contábeis, sobretudo dentro dos limites legais, que é o que caracteriza o gerenciamento de resultados.

Como sugestão para pesquisas futuras, registrase a aplicação a amostras com cooperativas de outros sistemas, ainda não pesquisados como os sistemas Unicred e Confessol, e mesmo com cooperativas de crédito não filiadas a algum sistema, chamadas independentes. Pois, a pesquisa sobre o gerenciamento de resultados está em curso a aproximadamente mais de duas décadas, muito tem sido aprendido, mas muitas questões interessantes permanecem sem resposta, assim a aplicação desta temática, seja trabalhando o gerenciamento a partir de decisões operacionais ou de decisões contábeis, torna-se um campo de pesquisa promissor. Assim como a discussão e aplicação de metodologias distintas para se captar estas estratégias que resultam no gerenciamento de resultados contábeis.

Por fim, ressalta-se que as cooperativas de crédito exercem um importante papel social, democratizando o acesso ao sistema financeiro nacional para agentes econômicos que carecem de produtos financeiros de qualidade, seja do ponto de vista da aplicação ou tomada de recursos. Espera-se que este 
estudo possam contribuir para a disseminação do tema gerenciamento de resultados, de forma a contribuir também no sentido de chamar a atenção para este tipo especial de instituição financeira que é a cooperativa de crédito.

\section{Referências}

AHMED, A. S.; TAKEDA, C.; THOMAS, S. Bank loan loss provisions: a reexamination of capital management, earnings management and signaling effects. Journal of Accounting \& Economics, v. 28,n. 1, p. 1-25. 1999.

BROWN, C.; DAVIS, K.. Capital management in mutual financial institutions. Journal of Banking \& Finance, vol. 33, n. 3, p 443-445, 2008.

BURGSTAHLER, D.; DICHEV, I. Earnings management to avoid earnings decrease and losses. Journal of Accounting \& Economics, NorthHolland, v.24, n.1, p.99-126, Dec. 1997.

DANTAS, J. A.; MEDEIROS, O. R.; GALDI, F. C.; COSTA, F. M.. Gerenciamento de resultados em bancos com uso de TVM: validação de modelo de dois estágios. Revista Contabilidade \& Finanças (Online), v. 24, p. 37-54, 2013.

DECHOW, P. M.; RICHARDSON, S. A.; TUNA, I. Why are earnings kinky? Na examination of the earnings management Explanation. Review of Accounting Studies, vol. 8, n. 2-3, p. 355-384, 2003.

DOORNIK, J. A.; HANSEN, H.. An Omnibus Test for Univariate and Multivariate Normality. Working Paper, Nuffield College, Oxford, 1994. p. 1-16. Disponível em: <www.doornik.com/ research/normal2.pd>f. Data de acesso: 13/08/2014.

GOULART, A. M. C. Gerenciamento de resultados contábeis em instituições financeiras no Brasil. 219 p. Tese (Doutorado em Ciências Contábeis) — FEA, Universidade de São Paulo, SP, 2007.

GUNNY, A. K. The relation between earnings management using real activities manipulation and future performance: Evidence from meeting earnings benchmarks. Contemporary Accounting Research, vol. 27,n. 3, p. 855-888, 2010.

HEALEY, P. M.; WAHLEN. J. M. A review of the earnings management literature and its implications for standard setting. Accounting Horizons, vol. 13,n. 4, p. 365-383, 1999.

HILLIER, D.; HODGSON, A.; STEVENSONCLARKE, P.; LHAOPADCHAN, S. Accounting Window Dressing and Template Regulation: A Case Study of the Australian Credit Union Industry. Journal of Business Ethics, v.83, n. 3, p. 579-593, 2008.

JACOB, John; JORGENSEN, Bjorn N. Earnings Management and accounting income aggregation. Journal of Accounting and Economics. $n$. 43, p. 369-390, Jan. 2007.

MAIA. S. C.; BRESSAN, V. G. F.; LAMOUNIER, W. M.; BRAGA, M. J. Gerenciamento de resultados em cooperativas de crédito no Brasil. Brazilian Busnisess Review. v.10, n.4, p,96-116, out.-dez, 2013.

MARTINEZ, A. L. Gerenciamento dos resultados contábeis: estudo empírico das companhias abertas brasileiras. 2001. $154 \mathrm{f}$. Tese (Doutorado em Ciências Contábeis) - FEA, Universidade de São Paulo, SP, 2001.

MARTINEZ, A. L. Gerenciamento de resultados no Brasil: um survey da literatura. Brazilian Business Review. v. 10, n. 4, p. 1-31, out-dez, 2013.

MATSUMOTO, A. S.; PARREIRA, E.M. Uma pesquisa sobre o Gerenciamento de Resultados Contábeis: causas e consequencias. UNB Contábil, vol. 10, n. 1., p.141-157, 2007.

MOREIRA, J. A. C. Manipulação para Evitar Perdas: o Impacto do Conservantismo. Faculdade de Economia da Universidade do Porto. May 2006. Disponível em: <http://www.fep.up.pt/investigacao/cete/papers/d p0605.pdf $>$. Data de acesso: 13/06/2014.

MOYER, S. E. Capital adequacy ratio regulations and accounting choices in commercial banks. 
Journal of Accounting \& Economics, vol 3, n. 2, p. 123-154. 1990.

PAULO E. Manipulação das informações contábeis: Uma análise teórica e empírica sobre os modelos operacionais de deteccão de gerenciamento de resultados. 2007. 269 f. Tese (Doutorado em Ciências Contábeis) - FEA, Universidade de São Paulo, SP, 2007.

\section{PORTAL DO COOPERATIVISMO DE CRÉDI-} TO. Dados consolidados dos Sistemas Cooperativos. Disponível em: $<$ http://cooperativismodecredito.coop.br/cenariobrasileiro/dados-consolidados-dos-sistemascooperativos/.> Data de acesso: 01/12/2014(a).

PORTAL DO COOPERATIVISMO DE CRÉDITO. Cooperativismo Financeiro amplia participação de mercado e atinge $5 \%$ do volume de depósitos do SFN. Disponível em: <http://cooperativismodecredito.coop.br/2014/11 /cooperativismo-financeiro-amplia-participacaode-mercado-e-atinge-5-do-volume-de-depositosdo-sfn/. > Data de acesso: 01/12/2014 (b).

PORTAL DO COOPERATIVISMO DE CRÉDITO. Sistema Sicredi. Disponível em: <http://cooperativismodecredito.coop.br/cenariobrasileiro/sistema-sicredi/ >Data de acesso: 01/12/2014 (c).

RODRIGUES, A.; PAULO, E.; CARVALHO, L. $\mathrm{N}$. Gerenciamento de resultados por meio das transações entre companhias brasileiras interligadas. RAUSP. Revista de Administração, v. 42, .2, p. 216-226. 2007.

REIS, E. M. Um estudo empírico do gerenciamento de resultados por meio de decisões operacionais nas empresas abertas brasileiras. 2014. $154 \mathrm{f}$. Dissertação (Mestrado em Ciências Contábeis) Faculdade de Ciências Econõmicas, Universidade Federal de Minas Gerais, MG, 2014.

RONEN, J.; YAARI, V. Earnings management: Emerging insights in theory, practice, and research. 1. Ed. New York: Springer. 2010.

ROSA, R. C.; TIRAS, S. L. Adoção do IFRS no Brasil: um terreno fértil para pesquisa sobre ge- renciamento de resultados. Brazilian Business Review. v. 10, n. 4, p. 141-156, out-dez, 2013.

VENTURA, E. C. F. (Org). Governança Cooperativa: Diretrizes e mecanismos para o fortalecimento da governança em cooperativas de crédito do Brasil. Brasília: BCB, 2009 Disponível em: < http://www.bcb.gov.br/Pre/microFinancas/coopc ar/pdf/livro_governanca_cooperativa_internet.pd f.>. Acesso em: 14/06/2014.

XAVIER, P. H. M.. Gerenciamento de resultados por bancos comerciais no Brasil.125f Tese (Doutorado em Controladoria e Contabilidade: Contabilidade) - FEA, Universidade de São Paulo, São Paulo, 2007.

ZENDERSKY, H. C. Gerenciamento de resultados em instituições financeiras no Brasil - 2000 a 2004. 131f. Brasília. Dissertação (Mestrado em Ciências Contábeis) - Programa Multiinstitucional e Inter-Regional de Pós-Graduação em Ciências Contábeis da Universidade de Brasília, Brasília, 2005.

* Os autores agradecem ao CNPq pelo apoio concedido para a realização desta pesquisa. 